\title{
Steatosis does not impair liver regeneration after partial hepatectomy
}

\author{
Svenja Sydor ${ }^{1}$, Yanli Gu², Martin Schlattjan¹, Lars P Bechmann¹, Ursula Rauen³, Jan Best ${ }^{1}$, Andreas Paul ${ }^{2}$, \\ Hideo A Baba ${ }^{4}$, Jan-Peter Sowa ${ }^{1}$, Guido Gerken ${ }^{1}$ and Ali Canbay ${ }^{1}$
}

Hepatic steatosis is a key feature of non-alcoholic fatty liver disease (NAFLD). While storage of lipid droplet-bound triglycerides in simple steatosis is physiologically inert, non-alcoholic steatohepatitis (NASH) is associated with hepatocyte damage and apoptosis. Mitochondrial oxidation of free fatty acids (FFA), derived from lipid droplets and hepatocellular uptake, is a rapid and effective way of energy supply for proliferating cells and FFA esterification provides substrates for lipid synthesis and cell proliferation. Thus, we investigated whether simple steatosis induced by western diet (WD) improves liver regeneration after partial hepatectomy (PHx). WD feeding for 6 weeks caused simple steatosis with hepatic lipid droplet and triglyceride accumulation accompanied by induction of fatty acid transport proteins (FATP), death receptors (DR), pro- and anti-apoptotic genes, hepatocyte growth factor $(\mathrm{Hgf})$ as well as increased serum leptin levels in a mouse model. After PHx, liver cell proliferation was higher in WD-fed mice and associated with FATP and Hgf induction. In addition, Erk1/2 (extracellular-related MAP kinase 1/2) dephosphorylation observed in standard diet (SD) mice was reduced in WD animals. PHx in steatotic livers did not affect hepatocyte apoptosis, despite DR upregulation. WD-induced steatosis enhances liver cell proliferation, which is accompanied by increased $\mathrm{Hgf}$ and leptin signaling as well as Erk1/2 phosphorylation. Induction of mild steatosis may therefore be beneficial for surgical outcome of hepatectomies. Laboratory Investigation (2013) 93, 20-30; doi:10.1038/labinvest.2012.142; published online 15 October 2012

KEYWORDS: fatty acid transport proteins; NAFLD; proliferation

Non-alcoholic fatty liver disease (NAFLD) and obesity are associated with chronically elevated levels of serum-free fatty acids (FFA), ${ }^{1,2}$ increased fatty acid uptake and lipid synthesis resulting in accumulation of triglycerides (TAG) within lipid droplets in hepatocytes. ${ }^{3}$ Insulin resistance and FFA abundance promote lipotoxicity, ROS formation and induce hepatic inflammation and hepatocyte apoptosis, which facilitates progression to non-alcoholic steatohepatitis (NASH). In addition, NASH patients show increased hepatic fatty acid transport protein (FATP) expression, which is associated with extrinsically and intrinsically induced apoptosis and death receptor (DR) upregulation. ${ }^{4}$ Thus, FFA-induced lipotoxicity rather than TAG accumulation in hepatocytes accounts for liver injury and susceptibility to liver damage. ${ }^{5}$ It is not clear, though, whether simple steatosis affects vulnerability of hepatocytes.

In cadaveric and living donor liver transplantation, organs with severe steatosis ( $>30 \%$ ) are excluded from liver transplantations, as steatohepatitis was identified as a significant risk factor for postoperative complications. ${ }^{6}$ The increasing prevalence of NAFLD and in particular NASH in prospective donors diminishes the availability of organs in future. ${ }^{7}$ Apparently $15-20 \%$ of the western population is obese, with up to $80-90 \%$ obese patients affected with NAFLD. Approximately 10-25\% of these NAFLD patients develop NASH, whereas about $2-3 \%$ of the general population is estimated to have NASH. ${ }^{8,9}$ However, some studies demonstrated that the risk for complications in transplantation utilizing organs with mild steatosis was overestimated and this may enlarge the pool of potential donor organs. ${ }^{10,11}$ Additionally, liver regeneration after partial hepatectomy (PHx) in mice and rats is associated with hepatic lipid accumulation and includes activation of inflammationrelated signaling molecules, such as interleukin 6 (IL-6), nuclear factor kappa $\mathrm{B}(\mathrm{nF} \kappa \mathrm{B})$ and signal transducer and

\footnotetext{
${ }^{1}$ Department of Gastroenterology and Hepatology, University Hospital Essen, Essen, Germany; ${ }^{2}$ Department of General, Visceral and Transplantation Surgery, University Hospital Essen, Essen, Germany; ${ }^{3}$ Institute of Physiological Chemistry, University Hospital Essen, Essen, Germany and ${ }^{4}$ Institute of Pathology and Neuropathology, University Hospital Essen, Essen, Germany

Correspondence: Professor A Canbay, MD, Department of Gastroenterology and Hepatology, University Hospital Essen, Hufelandstr. 55, Essen 45122, Germany. E-mail: ali.canbay@uni-due.de 
activator of transcription-3 (STAT3). ${ }^{12-15}$ Therefore, we aimed to investigate if steatosis alone (i) enhances vulnerability of the liver to damage, (ii) impairs proliferation and regeneration after $\mathrm{PHx}$ and (iii) which mechanisms are involved in this enhanced susceptibility.

The regenerative response and mRNA expression of FATP and DR in a model of liver regeneration in the steatotic liver were analyzed. In this model, hepatic TAG accumulation improved hepatocyte proliferation following PHx in mice, which was accompanied by induction of hepatocyte growth factor $(H g f)$ and enhanced postoperative Erk1/2 phosphorylation.

\section{MATERIALS AND METHODS Cell Preparation from Human Tissue and Sample Handling}

Liver tissue was obtained from patients undergoing resection for removal of liver tumors or resected segments from whole livers obtained from transplantation recipients. Cells were isolated from seven patients with liver resection due to various ethiologies. All patients gave written informed consent to this and all procedures conformed to the revised Declaration of Helsinki (Edinburgh 2000) and were reviewed by the ethics committee of the University Hospital Essen.

All cell preparations were conducted from macroscopically tumor-free material. For isolation of human primary hepatocytes, resected liver segments were perfused with HBSS (PAA, Pasching, Austria) with EGTA to wash out remaining blood followed by HBSS with $240-250 \mathrm{U} / \mathrm{ml}$ collagenase type IV (Sigma-Aldrich, Steinheim, Germany) to digest connective tissue. The obtained cell suspension was filtered through a $4-\mu \mathrm{m}$ mesh and washed three times in HBSS. The cell pellet was resuspended in cell culture medium (DMEM/ Ham's F-12 with $10 \%$ heat-inactivated FCS, $100 \mathrm{U} / \mathrm{ml}$ penicillin, $0.1 \mathrm{mg} / \mathrm{ml}$ streptomycin and $2 \mathrm{mM}$ L-glutamine; PAA, Pasching, Austria). To ascertain cell viability, cell suspension was stained by trypan blue and seeded at a viability of $95-99 \%$ and a density of $\sim 1 \times 10^{6} \mathrm{cells} / \mathrm{cm}^{2}$. At $1 \mathrm{~h}$ after seeding and periodically gently shaking culture medium was changed to remove non-adherent or non-attached as well as dead cells. On the next day, culture medium was changed again and 2 days after isolation, cells were used for performance of the experiment. For mimicking a steatosis-like state, cells were incubated for $24-96 \mathrm{~h}$ with 0.5 and $1 \mathrm{mM}$ mixed long-chain FFA, ie, 2:1 oleate: palmitate (Sigma-Aldrich). Controls were kept without FFA. Total RNA from cultured cells was isolated with the RNeasy Mini kit (Qiagen, Hilden, Germany). To obtain total protein extracts, hepatocytes were lysed for $30 \mathrm{~min}$ at $4{ }^{\circ} \mathrm{C}$ in lysis buffer $(50 \mathrm{mM}$ Tris- $\mathrm{HCl}$, $\mathrm{pH}$ 7.5, $150 \mathrm{mM} \mathrm{NaCl}$, plus protease inhibitors). Lysates were centrifuged at $13000 \mathrm{~g}$ for $30 \mathrm{~min}$ at $4{ }^{\circ} \mathrm{C}$. Protein concentrations were determined by BCA assay (Pierce, Bonn, Germany).

\section{Animals and Surgical Procedures}

Four- to six-week-old $\mathrm{C} 57 \mathrm{Bl} / 6$ mice were fed a standard ( $n=4$ /group) or western $\operatorname{diet}(\mathrm{WD}) \quad(n=6 /$ group $)$
(TD.88137, Harlan; Madison WI, USA) for 6 weeks. Before surgical intervention, animals were anesthetized, $70 \% \mathrm{PHx}$ was performed by removing the left and median lobes of the liver. ${ }^{16}$ Mice were killed on postoperative days 1, 2 and $7(\mathrm{PHx}+1, \mathrm{PHx}+2$ and $\mathrm{PHx}+7)$. Total blood was drawn from the vena cava, centrifuged and serum was stored at $-80^{\circ} \mathrm{C}$. Total protein, albumin, total bilirubin, glucose, AST, ALT and LDH were measured using Spotchem II-system (Akray; Kyoto, Japan). All mice were bred and housed in the animal facility of the University Hospital Essen (ZTL), University of Duisburg-Essen, Germany according to the recommendations of the Federation of European Laboratory Animal Science Association (FELASA). All procedures were approved by the Landesamt für Natur, Umwelt und Verbraucherschutz Nordrhein-Westfalen (LANUV NRW).

\section{Histopathology and Sample Handling}

Liver tissue was stored in $4.5 \%$ formalin solution, paraffin embedded and sectioned. Samples for Oil-Red-O staining were embedded in Tissue-Tek OCT Compound cryo medium (Sakura Finetec Europe; Alphen aan den Rijn, Netherlands) and sectioned. Stainings were performed using standard protocols; Ki67-antibody was purchased from DCS Biogenex (Hamburg, Germany), phospho histone $\mathrm{H} 3$-antibody was purchased from Abcam (Cambridge, UK), F4/80-antibody was purchased from AbD Serotec (Oxford, UK). TUNEL staining was performed using In Situ Cell Death Detection Kit Flourescein from Roche (Mannheim, Germany). Quantification of Oil-Red-O staining was performed using Axiovision 4.8 (Carl Zeiss, Jena, Germany). Liver tissue for RNA and protein isolation was frozen in liquid nitrogen. Total RNA was isolated by TRIzol extraction (Invitrogen, Darmstadt; Germany) and purified utilizing RNeasy Mini Kit (Qiagen). Reverse transcription was performed with the QuantiTect RT kit (Qiagen) using $1 \mu \mathrm{g}$ of total RNA.

\section{Quantitative Real-Time PCR}

Gene expression levels were measured by quantitative real-time PCR using succinate dehydrogenase complex subunit A (Sdha) as housekeeping gene; primer sequences are given in Supplementary Table S1.

\section{Western Blotting}

Protein lysates were prepared using lysis buffer $(50 \mathrm{mM}$ Tris-HCl; $150 \mathrm{nM} \mathrm{NaCl} ; 0.1 \% \mathrm{NP}-40 ; 1 \%$ desoxycholic acid) containing complete mini EDTA-free protease inhibitor cocktail and phosphostop (Roche, Mannheim, Germany). In all, $30 \mu \mathrm{g}$ of total protein were separated using SDS-PAGE, immunoblotting was performed using standard procedures with the following primary antibodies from Cell Signaling Technology (Danver, MA, USA): phospho-p44/42 MAPK (Erk1/2) (Thr202/Tyr204) (197G2); $\beta$-Actin (13E5). 


\section{Biochemical Assays and ELISAs}

Biochemical assays for TAG and FFA in serum or tissue were performed using Triglyceride Quantification Kit and Fatty Acid Quantification Kit from Biovision/BioCat GmbH (Heidelberg, Germany). Serum leptin was determined using Bio-Plex Pro Diabetes Assay (Bio-Rad, Munich, Germany). Adiponectin serum levels were quantified using mouse adiponectin/Acrp30 Quantikine Kit from R\&D Systems (Minneapolis, MN, USA). Serum cytokines and chemokines were determined using Bio-Plex Pro Mouse Cytokine, Chemokines and Growth Factors Assay (Bio-Rad). Hepatic ATP levels were measured using ATP bioluminescent somatic cell assay kit from Sigma (St Louis, MO, USA). The apoptosis marker M30 was assessed in protein lysates from cultured hepatocytes using the M30Apoptosense ELISA kit (Peviva, Bromma, Sweden).

\section{Statistical Analysis}

Statistical significance was determined using an unpaired (or paired, when applicable), two-tailed $t$-test, performed with GraphPad Prism 5 (GraphPad Software, La Jolla, CA, USA). Significance was assumed at $P \leqslant 0.05$. If not stated otherwise, all data is presented as mean \pm s.e.m.

Relative gene expressions were calculated from the threshold cycles in relation to the housekeeping gene and to untreated controls.

\section{RESULTS}

\section{Upregulation of Fatty Acid-Binding Protein 1 and DRs} in Steatotic Hepatocytes does not Induce Apoptosis

To evaluate the effect of FFA on human primary hepatocytes, the mRNA expression of FATP and DR was quantified after incubation with FFA for 24-96 h. Fatty acid-binding protein 1 (FABP-1) expression was induced after treatment with FFA for 24,48 and $72 \mathrm{~h}$ (Figure 1a). The treatment with $1 \mathrm{mM}$ FFA did not affect the expression of TNFR1, whereas after $24 \mathrm{~h}$, incubation with $0.5 \mathrm{mM}$ FFA TNFR1-expression was increased (Figure 1b). The treatment with $1 \mathrm{mM}$ FFA merely activated CD95/Fas-expression after 24, 48 and $72 \mathrm{~h}$ but incubation with $0.5 \mathrm{mM}$ FFA activated the expression of CD95/Fas after 24, 48, 72 and 96 h (Figure 1c). Surprisingly, the incubation with FFA for $24-96 \mathrm{~h}$ with or without the CD95/Fas-agonist CH11 did not affect apoptosis induction in primary human hepatocytes, as shown by measurement of cleaved cytoceratin-18 fragments by M30-ELISA in cell lysates of human primary hepatocytes (Figure 1d).

\section{Hepatic Steatosis Induces Fatty Acid Transporters, Pro- and Anti-Apoptotic Genes and Hgf in Mice}

As we did not observe enhanced apoptosis or cellular damage in vitro, we tested liver cell damage in an in vivo model of steatosis. HE staining showed increased lipid droplet accumulation of a microvesicular type in hepatocytes in WD animals (Figure 2a). Serum FFA and TAG in liver tissue were significantly elevated after 6 weeks of WD compared with standard diet (SD) mice (Table 1). F4/80 immunostaining showed augmented agglomeration of activated macrophages in liver tissue of WD animals indicating a slight tissue inflammation after 6 weeks of WD (Figure 2b). Additional ASDCL staining to designate neutrophil granulocytes did not show noticeable differences between the two feeding groups (Supplementary Figure 1). Fibrotic collagen deposition, visualized by Sirius-Red staining, was not found in SD or WD livers (Supplementary Figure 2). Serum AST, ALT, LDH and leptin were slightly increased after 6 weeks WD, whereas bilirubin, serum protein, albumin and adiponectin remained unchanged (Table 1). These findings reveal that feeding WD
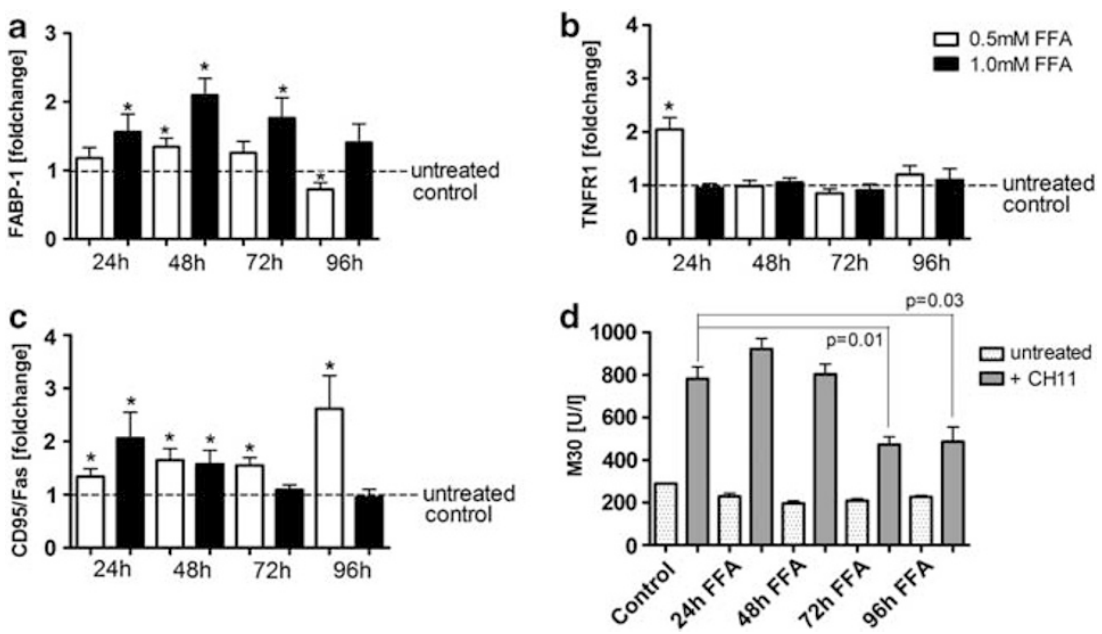

Figure 1 mRNA expression of CD95/Fas, TNFR1 and fatty acid-binding protein (FABP)-1 as well as apoptosis marker M30 in human primary hepatocytes after free fatty acids (FFA) treatment. FFA incubation for 24-96 h increased CD95/Fas and FABP-1 expression in primary hepatocytes (a and c) but did not alter vulnerability to CD95/Fas-induced cell death shown by measurement of apoptosis marker M30 (d). TNFR1 expression was activated only after stimulation with $0.5 \mathrm{mM}$ FFA for $24 \mathrm{~h}$ (b). PCR data represent relative expression vs untreated cells (fold change) as mean \pm s.e.m. of $n=7,{ }^{*} P<0.05$ vs untreated control cells. Quantification of M30 shown as mean \pm s.e.m. of $n=3$. 

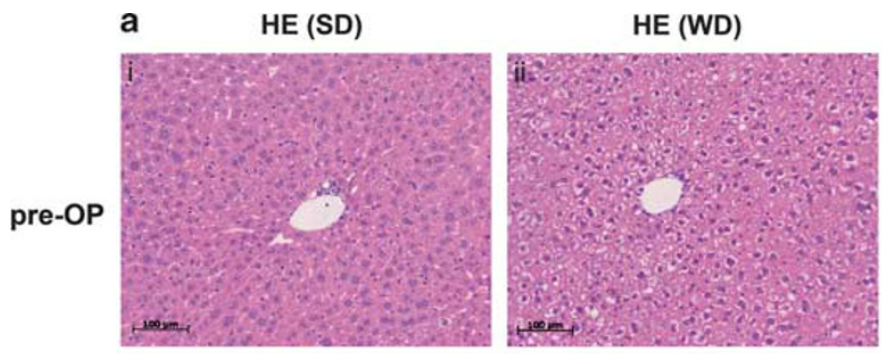

b
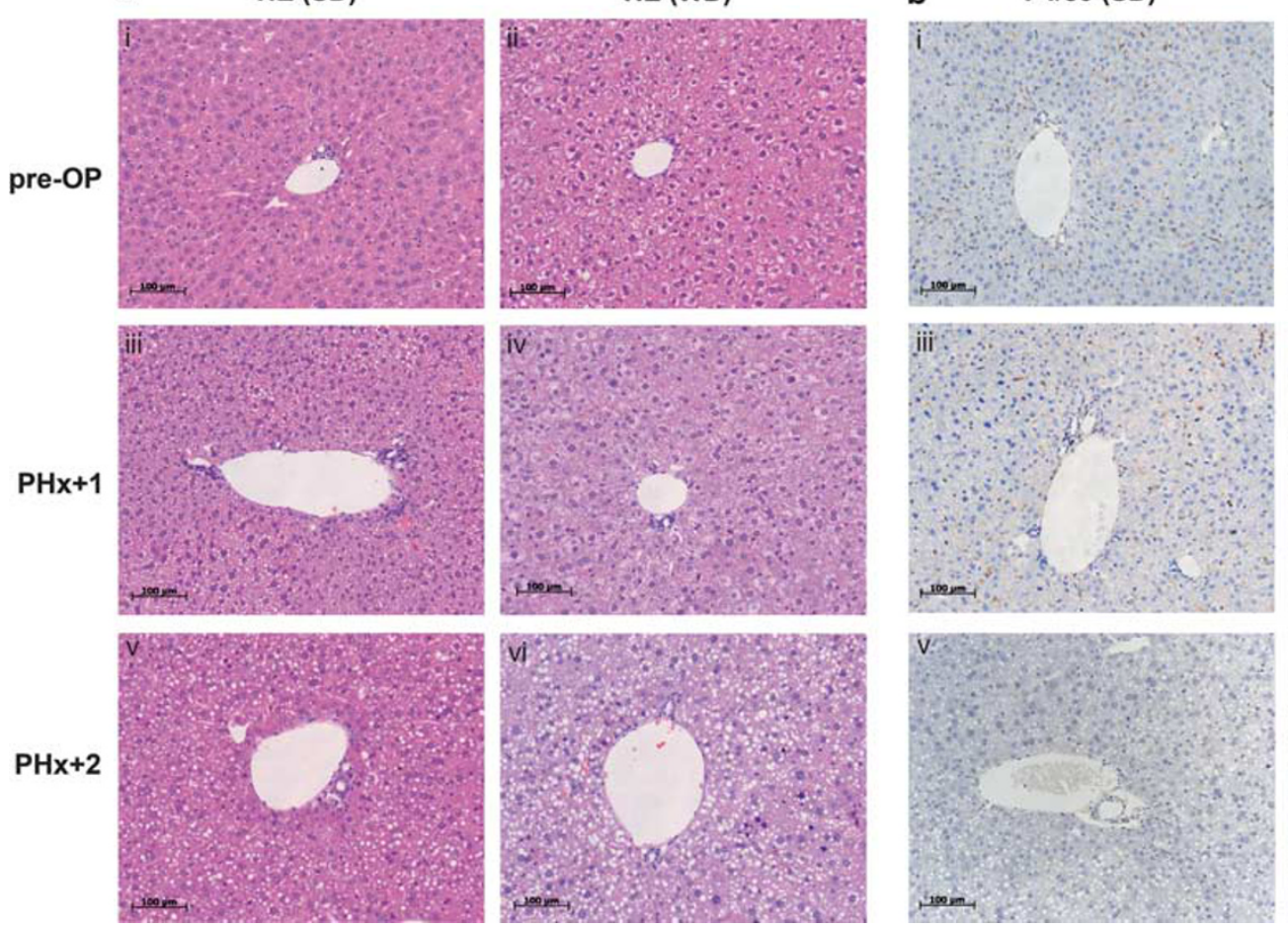
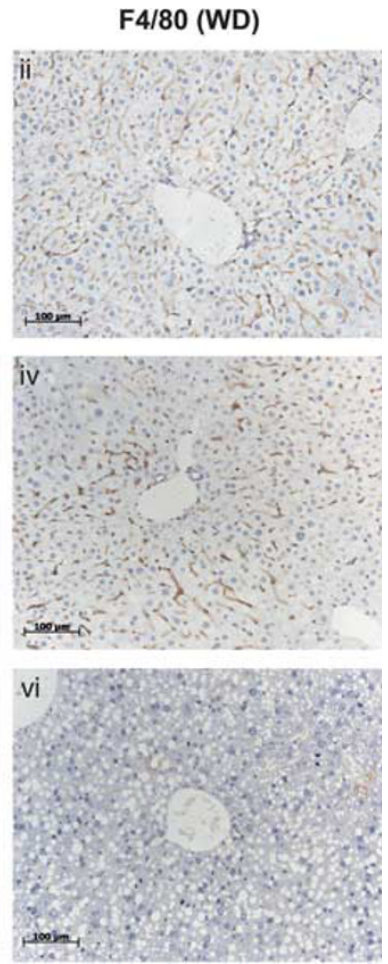

Figure $2 \mathrm{HE}-$ and F4/80 staining of liver tissue from mice before and after 70\% hepatectomy. HE staining (a) of liver tissue from mice with standard diet (SD) and western diet (WD) demonstrated solid accumulation of fat droplets of microvesicular type after 6 weeks of WD feeding. F4/80 staining (b) did not show altered inflammation comparing SD- and WD-fed animals. Panels i, iii and $v$ show representative images of SD-fed mice, panels ii, iv and vi of WD-fed mice. Panels $\mathrm{i}$ and ii show images before $70 \%$ hepatectomy (pre-OP), iii and iv on postoperative day 1 (PHx +1$)$, v and vi show images of liver tissue on postoperative day $2(\mathrm{PHx}+2)$; 200-fold magnification.

for 6 weeks impact liver histology and suggest modest liver injury. These metabolic features resemble those found in most overweight patients, though pro-inflammatory serum cytokines and chemokines were not changed significantly in WD-fed animals (Table 2).

As previously shown by our group in steatotic patients and others in rats, ${ }^{1,17}$ expression levels of Fabp-1 and Fatp-5 were significantly upregulated in WD-fed mice (Figure $3 \mathrm{~b}$ and c). Cd36/Fat expression was slightly increased in WD-fed mice (Figure 3a). Expression levels of Ppara and corresponding target genes Acoxl and $P d k 1$ were not changed (Figure $3 \mathrm{e}-\mathrm{g}$ ). Ppar $\alpha$ has a crucial role within FFA and TAG metabolism and its target genes Acoxl and $P d k 1$ catalyze fat and carbohydrate degradation.

DR-mRNA expression (Figure $4 \mathrm{a}$ and $\mathrm{b}$ ) and the proapoptotic molecule Noxa (Figure 4c) were upregulated in steatotic livers. Interestingly, expression levels of anti-apoptotic molecules (Figure $4 \mathrm{~d}-\mathrm{f}$ ) and the regeneration-associated factor $\mathrm{Hgf}$ (Figure 3d) were also elevated after 6 weeks of WD feeding. To elucidate the susceptibility of the liver to apoptosis, the ratio of anti-apoptotic $\mathrm{Bcl}-2$ expression to proapoptotic Bax expression levels was determined (Figure 4g). Before $\mathrm{PHx}$, this ratio was slightly elevated in WD-fed animals. After PHx, no significant differences were seen. To evaluate if the functional effects of pro- and anti-apoptotic signaling pathways as well as a slight tissue inflammation alter hepatocellular death, a TUNEL assay was performed. Apoptosis was not increased in liver tissue of WD-fed mice (Figure $4 \mathrm{~h}$ ), suggesting that WD-induced DR upregulation is compensated by induction of anti-apoptotic genes in mice with simple steatosis.

\section{WD Induces Hepatocyte Proliferation after PHx}

To elucidate the impact of WD-induced steatosis on liver regeneration, 70\% PHx was performed. The preoperatively elevated serum FFA were maintained in WD-fed mice, though an increase of FFA in SD animals neutralized the differences between SD and WD after 70\% PHx (Table 1). TAG and leptin levels were significantly elevated in liver tissue of WD-fed mice before and on $\mathrm{PHx}+1,2$ and 7 (Table 1). On $\mathrm{PHx}+1$, serum parameters for liver injury (ALT, AST, LDH) were elevated (Table 1), with a stronger increase in SD-fed mice. On PHx +2 and +7 , AST, ALT and LDH concentrations were marginally elevated. In pre-OP livers of SD-fed mice, no obvious fat droplets were found, though HE (Figure 2a) and Oil-Red-O staining (not shown) revealed enhanced fat accumulation in liver tissue on $\mathrm{PHx}+1$ and $\mathrm{PHx}+2$. This effect was also seen in WD-fed animals with further lipid droplet accumulation in the liver, leading to no observable differences between SD and WD on 
Table 1 Serum-derived parameters and liver triglycerides of animals fed a standard (SD) or western diet (WD) before PHx (pre-OP) or on postoperative days 1, 2 and 7 (PHx +)

\begin{tabular}{|c|c|c|c|c|}
\hline Parameter & $\begin{array}{l}\text { Time } \\
\text { point }\end{array}$ & SD & WD & $P$-value ${ }^{a}$ \\
\hline \multirow[t]{4}{*}{ FFA (nmol) } & Pre-OP & $5.3 \pm 0.21$ & $9.85 \pm 0.14$ & $<0.0001^{\mathrm{a}}$ \\
\hline & $\mathrm{PHx}+1$ & $9.05 \pm 0.66$ & $9.26 \pm 0.47$ & n.s. \\
\hline & $\mathrm{PHx}+2$ & $8.19 \pm 1.16$ & $8.81 \pm 0.65$ & n.s. \\
\hline & $\mathrm{PHx}+7$ & $8.78 \pm 0.64$ & $9.04 \pm 0.61$ & n.s. \\
\hline Triglycerides (liver & Pre-OP & $41.27 \pm 5.60$ & $78.98 \pm 8.52$ & $0.0008^{\mathrm{a}}$ \\
\hline \multirow[t]{3}{*}{ tissue) ( $\mu \mathrm{mol} / \mathrm{mg}$ ) } & $\mathrm{PHx}+1$ & $32.93 \pm 7.09$ & $99.27 \pm 16.36$ & $0.04^{a}$ \\
\hline & $\mathrm{PHx}+2$ & $63.17 \pm 1.44$ & $113.7 \pm 18.26$ & $0.03^{\mathrm{a}}$ \\
\hline & $\mathrm{PHx}+7$ & $25.05 \pm 11.85$ & $103.3 \pm 19.52$ & $0.03^{a}$ \\
\hline \multirow[t]{4}{*}{ AST (IU/I) } & Pre-OP & $26.75 \pm 8.0$ & $46.25 \pm 13.7$ & n.s. \\
\hline & $\mathrm{PHx}+1$ & $4480.0 \pm 18$ & $2038.0 \pm 169.3$ & n.s. \\
\hline & $\mathrm{PHx}+2$ & $113.0 \pm 10.54$ & $114.7 \pm 16.88$ & n.s. \\
\hline & $\mathrm{PHx}+7$ & $68.33 \pm 33.22$ & $72.83 \pm 28.78$ & n.s. \\
\hline \multirow[t]{4}{*}{ ALT (IU/I) } & Pre-OP & $17.5 \pm 4.91$ & $63.0 \pm 29.85$ & n.s. \\
\hline & $\mathrm{PHx}+1$ & $2269.0 \pm 389.1$ & $1099.0 \pm 154.4$ & $0.012^{\mathrm{a}}$ \\
\hline & $\mathrm{PHx}+2$ & $99.33 \pm 8.69$ & $68.33 \pm 9.04$ & n.s. \\
\hline & $\mathrm{PHx}+7$ & $90.0 \pm 56.87$ & $47.17 \pm 31.34$ & n.s. \\
\hline \multirow[t]{4}{*}{ LDH (IU/I) } & Pre-OP & $732.5 \pm 201.1$ & $2002.0 \pm 161.7$ & n.s. \\
\hline & $\mathrm{PHx}+1$ & $30610.0 \pm 3676.0$ & $18201.0 \pm 2311.0$ & $0.02^{a}$ \\
\hline & $\mathrm{PHx}+2$ & $2319.0 \pm 559.1$ & $1368.0 \pm 374.1$ & n.s. \\
\hline & $\mathrm{PHx}+7$ & $2344.0 \pm 832.2$ & $2578.0 \pm 345.4$ & n.s. \\
\hline \multirow[t]{4}{*}{ T-protein (g/dl) } & Pre-OP & $4.13 \pm 0.08$ & $4.33 \pm 0.21$ & n.s. \\
\hline & $\mathrm{PHx}+1$ & $3.73 \pm 0.10$ & $3.67 \pm 0.15$ & n.s. \\
\hline & $\mathrm{PHx}+2$ & $3.03 \pm 0.38$ & $1.97 \pm 0.18$ & n.s. \\
\hline & $\mathrm{PHx}+7$ & $3.73 \pm 0.18$ & $3.48 \pm 1.13$ & n.s. \\
\hline \multirow[t]{4}{*}{ Albumin (g/dl) } & Pre-OP & $2.0 \pm 0.06$ & $1.33 \pm 0.33$ & n.s. \\
\hline & $\mathrm{PHx}+1$ & $1.85 \pm 0.09$ & $1.92 \pm 0.06$ & n.s. \\
\hline & $\mathrm{PHx}+2$ & $1.25 \pm 0.25$ & $1.0 \pm 0.04$ & n.s. \\
\hline & $\mathrm{PHx}+7$ & $2.0 \pm 0.06$ & $1.67 \pm 0.07$ & n.s. \\
\hline \multirow[t]{4}{*}{ Bilirubin (mg/dl) } & Pre-OP & $0.48 \pm 0.08$ & $0.3 \pm 0.04$ & n.s. \\
\hline & $\mathrm{PHx}+1$ & $2.05 \pm 0.92$ & $2.17 \pm 0.82$ & n.s. \\
\hline & $\mathrm{PHx}+2$ & $0.3 \pm 0.06$ & $0.37 \pm 0.08$ & n.s. \\
\hline & $\mathrm{PHx}+7$ & $0.47 \pm 0.07$ & $0.38 \pm 0.03$ & n.s. \\
\hline \multirow[t]{4}{*}{ Leptin (pg/ml) } & Pre-OP & $983.4 \pm 170.9$ & $36416.0 \pm 21377.0$ & n.s. \\
\hline & $\mathrm{PHx}+1$ & $5242.0 \pm 1042.0$ & $17260.0 \pm 2431.0$ & $0.01^{a}$ \\
\hline & $\mathrm{PHx}+2$ & $1087.0 \pm 355.7$ & $6717.0 \pm 695.4$ & $0.002^{\mathrm{a}}$ \\
\hline & $\mathrm{PHx}+7$ & $975.5 \pm 190.1$ & $4650.0 \pm 412.6$ & $0.001^{a}$ \\
\hline \multirow[t]{4}{*}{ Adiponectin (ng/ml) } & Pre-OP & $2.72 \pm 0.84$ & $2.11 \pm 1.19$ & n.s. \\
\hline & $\mathrm{PHx}+1$ & $4.06 \pm 0.51$ & $5.44 \pm 0.75$ & n.s. \\
\hline & $\mathrm{PHx}+2$ & $1.98 \pm 0.42$ & $1.96 \pm 0.61$ & n.s. \\
\hline & $\mathrm{PHx}+7$ & $4.27 \pm 0.94$ & $3.02 \pm 0.68$ & n.s. \\
\hline
\end{tabular}

${ }^{\text {a }} P$-value is given for SD vs WD for each timepoint. n.s.: not significant.
Table 2 Serum-derived cytokines and chemokines of animals fed a standard (SD) or western diet (WD) before PHx (pre-OP) or on postoperative days 1, 2 and $7(\mathrm{PHx}+)$

\begin{tabular}{|c|c|c|c|c|}
\hline $\begin{array}{l}\text { Cyto-/chemokine } \\
\text { (pg/ml) }\end{array}$ & Time point & SD & WD & $P$-value ${ }^{a}$ \\
\hline \multirow[t]{4}{*}{ IL-1a } & Pre-OP & $94.9 \pm 57$ & $50.6 \pm 24.9$ & n.s. \\
\hline & $\mathrm{PHx}+1$ & $141.6 \pm 51.9$ & $69.7 \pm 29.6$ & n.s. \\
\hline & $\mathrm{PHx}+2$ & $163.5 \pm 41.6$ & $34.8 \pm 28.1$ & $0.03^{\mathrm{a}}$ \\
\hline & $\mathrm{PHx}+7$ & $32.9 \pm 26.2$ & $76.2 \pm 30.8$ & n.s. \\
\hline \multirow[t]{4}{*}{$\mid L-1 b$} & Pre-OP & $261.4 \pm 24.4$ & $209.5 \pm 21.2$ & n.s. \\
\hline & $\mathrm{PHx}+1$ & $238.2 \pm 16.3$ & $208.2 \pm 15.9$ & n.s. \\
\hline & $\mathrm{PHx}+2$ & $191.0 \pm 32.1$ & $191.0 \pm 9.7$ & n.s. \\
\hline & $\mathrm{PHx}+7$ & $270.1 \pm 97.3$ & $231.9 \pm 33$ & n.s. \\
\hline \multirow[t]{4}{*}{ IL-6 } & Pre-OP & $29.2 \pm 10.7$ & $23.0 \pm 12.8$ & n.s. \\
\hline & $\mathrm{PHx}+1$ & $86.0 \pm 29.2$ & $92.7 \pm 28.0$ & n.s. \\
\hline & $\mathrm{PHx}+2$ & $51.9 \pm 14.5$ & $18.0 \pm 3.8$ & n.s. \\
\hline & $\mathrm{PHx}+7$ & $36.8 \pm 14.3$ & $26.1 \pm 6.7$ & n.s. \\
\hline \multirow[t]{4}{*}{ IL-12 (p40) } & Pre-OP & $142.1 \pm 78.4$ & $116.9 \pm 41.6$ & n.s. \\
\hline & $\mathrm{PHx}+1$ & $139.2 \pm 48.0$ & $79.5 \pm 22.7$ & n.s. \\
\hline & $\mathrm{PHx}+2$ & $101.6 \pm 18.5$ & $77.1 \pm 27.3$ & n.s. \\
\hline & $\mathrm{PHx}+7$ & $154.3 \pm 20.0$ & $175.7 \pm 49.4$ & n.s. \\
\hline \multirow[t]{4}{*}{ IL-12 (p70) } & Pre-OP & $92.9 \pm 48.2$ & $47.0 \pm 21.3$ & n.s. \\
\hline & $\mathrm{PHx}+1$ & $130.4 \pm 47.9$ & $48.5 \pm 21.8$ & n.s. \\
\hline & $\mathrm{PHx}+2$ & $143.5 \pm 37.6$ & $41.8 \pm 22.8$ & $0.046^{\mathrm{a}}$ \\
\hline & $\mathrm{PHx}+7$ & $107.0 \pm 71.3$ & $104.8 \pm 32.8$ & n.s. \\
\hline \multirow[t]{4}{*}{ MCP-1 } & Pre-OP & $13.8 \pm 0.31$ & $69.6 \pm 37.0$ & n.s. \\
\hline & $\mathrm{PHx}+1$ & $230.5 \pm 85.5$ & $137.1 \pm 19.8$ & n.s. \\
\hline & $\mathrm{PHx}+2$ & $114.7 \pm 87.6$ & $56.8 \pm 27.0$ & n.s. \\
\hline & $\mathrm{PHx}+7$ & $238.7 \pm 224.8$ & $104.2 \pm 90.4$ & n.s. \\
\hline \multirow[t]{4}{*}{ TNF- $\alpha$} & Pre-OP & $45.9 \pm 3.1$ & $28.7 \pm 9.9$ & n.s. \\
\hline & $\mathrm{PHx}+1$ & $79.1 \pm 38.9$ & $53.4 \pm 14.7$ & n.s. \\
\hline & $\mathrm{PHx}+2$ & $39.4 \pm 18.3$ & $31.8 \pm 13.5$ & n.s. \\
\hline & $\mathrm{PHx}+7$ & $317.7 \pm 80.8$ & $125.3 \pm 80.6$ & n.s. \\
\hline \multirow[t]{4}{*}{ Rantes } & Pre-OP & $52.1 \pm 2.7$ & $32.2 \pm 12.5$ & n.s. \\
\hline & $\mathrm{PHx}+1$ & $119.6 \pm 55.2$ & $58.4 \pm 27.7$ & n.s. \\
\hline & $\mathrm{PHx}+2$ & $63.8 \pm 15.4$ & $27.6 \pm 12.3$ & n.s. \\
\hline & $\mathrm{PHx}+7$ & $48.9 \pm 17.2$ & $52.5 \pm 18.7$ & n.s. \\
\hline
\end{tabular}

${ }^{\mathrm{a}} P$-value is given for SD vs WD for each timepoint. n.s.: not significant.

$\mathrm{PHx}+1$ and $\mathrm{PHx}+2$ (Figure 2a). One week $(\mathrm{PHx}+7)$ after $70 \% \mathrm{PHx}$, there were no obvious fat droplets in the liver of SD-fed mice, whereas WD animals retained a substantial fat content.

Ki67 staining showed increased liver cell proliferation in WD mice after PHx (Figure 5a and b). On PHx +1 , only few proliferating cells were detected in WD-fed mice and almost no proliferating cells in SD-fed mice. In line with previous 
a

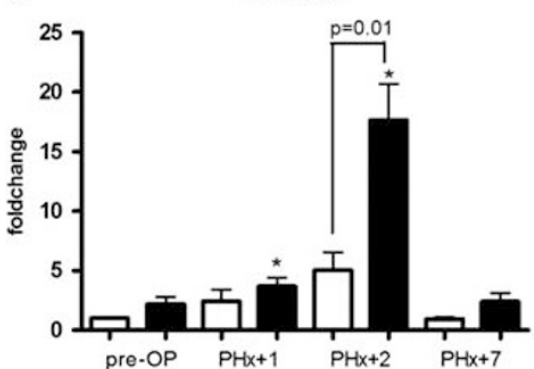

C

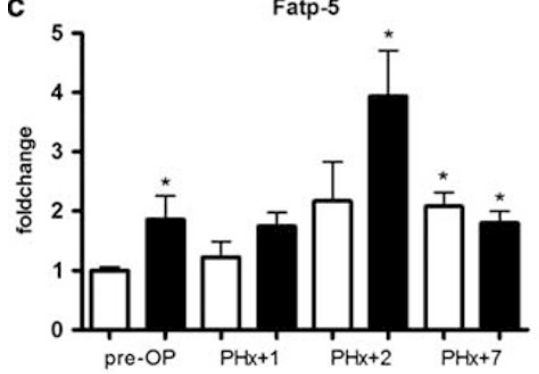

e

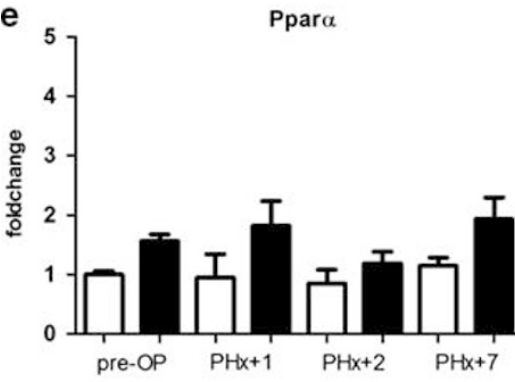

b

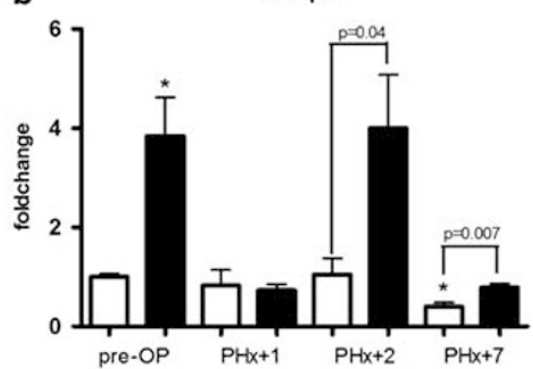

d

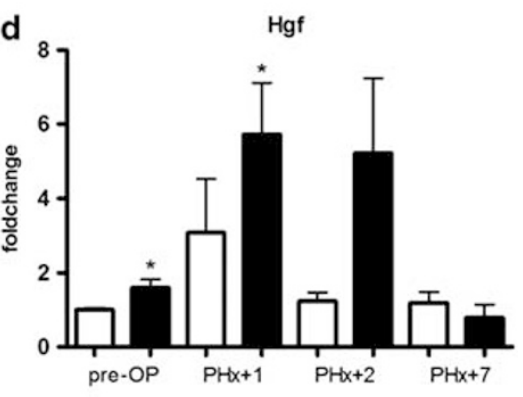

f 5 Acox 1

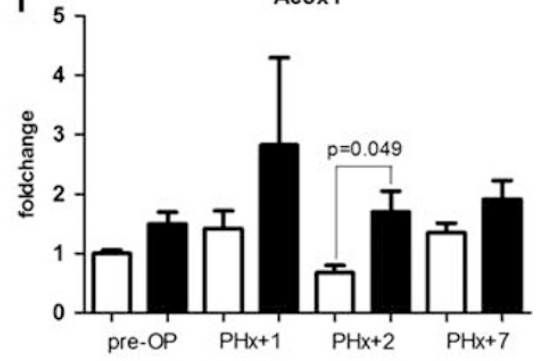

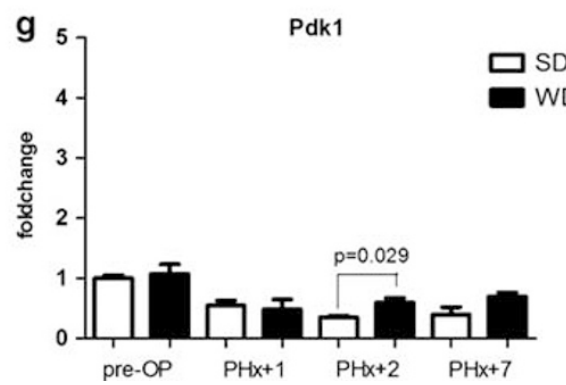

Figure 3 mRNA expression of fatty acid transport proteins in liver tissue of mice with dietary steatosis after $70 \%$ hepatectomy. Increased expression of Cd36/Fat (a) was observed on postoperative days $(\mathrm{PHx}+) 1$ and 2. Fatty acid-binding protein (Fabp-1) expression (b) was elevated on $\mathrm{PHx}+2$ and Fatp-5 expression (c) on $\mathrm{PHx}+2$ and 7. Expression of the crucial regenerative factor hepatocyte growth factor (Hgf) (d) was increased on PHx +1 in standard diet (SD)- and on $\mathrm{PHx}+1$ and 2 in western diet (WD)-fed mice. Expression of the fat-metabolism-related transcription factor Ppar $\alpha$ (e) was not changed significantly, Acox1 (f) and Pdk1 (g) (both target genes of Ppar $\alpha$ ) were elevated on PHx +2 in WD-fed animals. Data represent relative expression vs SD-fed animals (fold change) as mean \pm s.e.m., ${ }^{*} P<0.05$ vs SD pre-OP. PHx: $70 \%$ hepatectomy.

studies, highest proliferation rates were observed in SD- as well as WD-fed mice on $\mathrm{PHx}+2$, whereas there were significantly more proliferating cells in WD liver tissue (Figure 5b). Slightly reduced numbers of Ki67-positive cells were found in WD livers and even lower cell numbers in SD animals on $\mathrm{PHx}+7$. Mitosis rate was determined by phospho histone $\mathrm{H} 3$ staining (Figure 5c) and WD-fed mice showed more mitotic cells on $\mathrm{PHx}+2$ and $\mathrm{PHx}+7$ compared with SD-fed animals.
On $\mathrm{PHx}+1$, there were no phospho histone $\mathrm{H} 3$-positive cells in liver tissue of both groups.

\section{Hepatocyte Proliferation after PHx is Associated with Cd36/Fat and Hgf Induction}

As both groups exhibited a further increase of fat content in the liver, involvement of FATP was analyzed. Indeed, $70 \% \mathrm{PHx}$ resulted in a significant upregulation of Cd36/Fat 

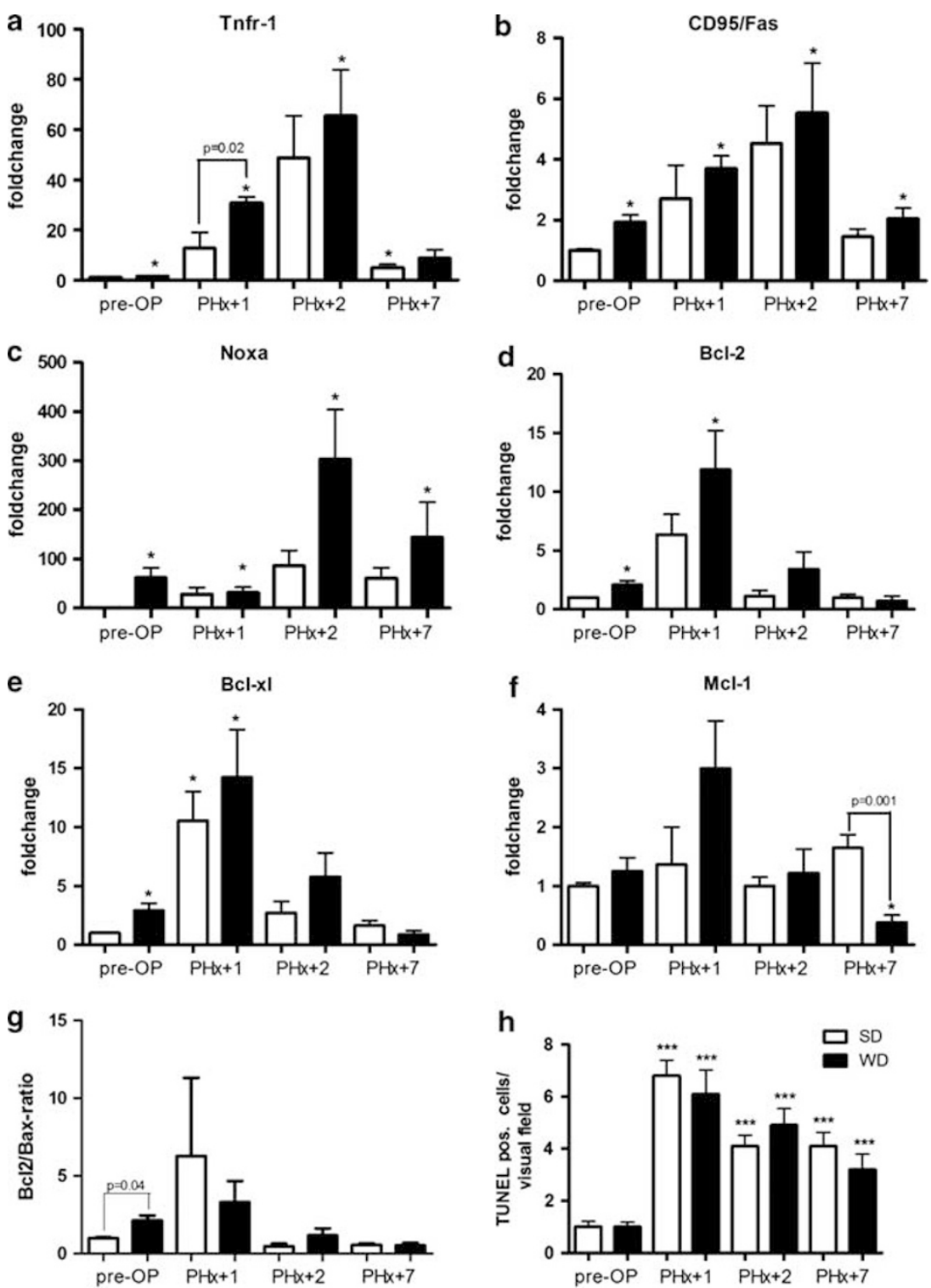

Figure 4 Cell death and apoptosis-related gene expression in steatotic murine liver tissue after $70 \%$ hepatectomy. Partial hepatectomy (PHx) led to increased death receptor (DR) expression (a, b) in liver tissue of standard diet (SD)- and western diet (WD)-fed mice on postoperative days (PHx +) 1, 2 and 7, which was more prominent in WD animals. A similar effect of PHx was present for Noxa expression (pro-apoptotic, c). mRNA expression of the anti-apoptotic molecules $\mathrm{BCl}-2$ (d) and $\mathrm{BCl}-\mathrm{xl}$ (e) were elevated on $\mathrm{PHx}+1$ and 2 in both groups, whereas $M c l-1$ expression (f) was only raised on $\mathrm{PHx}+1$. Determination of $\mathrm{BCl}-2 / \mathrm{Bax}$ ratio showed a surplus of anti-apoptotic $\mathrm{BCl}-2$ expression (g). Data of mRNA expression are depicted as mean \pm s.e.m. of the relative expression vs SD-fed animals (fold change), ${ }^{*} P<0.05$ vs SD pre-OP $(\mathbf{a}-\mathbf{g})$. Cell death was quantified by TUNEL staining, though no differences between SD and WD feeding were observable. Shown are TUNEL-positive cells per visual field as mean \pm s.e.m. ${ }^{* * *} P<0.001$ vs SD pre-OP (Figure $3 \mathrm{~h}$ ).

in WD-fed animals on $\mathrm{PHx}+1$ and $\mathrm{PHx}+2$ and only a slight increase on $\mathrm{PHx}+7$ (Figure 3a) compared with preoperative levels. This effect was less pronounced in SD-fed mice on $\mathrm{PHx}+1$ and $\mathrm{PHx}+2$. Expression of Fatp-5 was further elevated in WD animals on $\mathrm{PHx}+2$ and to a lower extent on PHx +7 (Figure 3c). Fabp-1 expression was increased on $\mathrm{PHx}+2$ in $\mathrm{WD}$ animals, although this did not reach significance (Figure 3b). Metabolic factors, such as
Ppara, Acoxl and Pdk1, were not changed on $\mathrm{PHx}+1$ and PHx +7 ; solely mRNA expression of Acoxl and Pdk1 were slightly elevated on $\mathrm{PHx}+1$ (Figure $3 \mathrm{e}-\mathrm{g}$ ).

Enhanced expression of $\mathrm{Hgf}$ was detected on $\mathrm{PHx}+1$ in both dietary groups with a stronger increase in WD-fed mice (Figure 3d), though the difference was not significant. In WD animals, this elevation continued until $\mathrm{PHx}+2$, which did not occur in the SD group. 
a
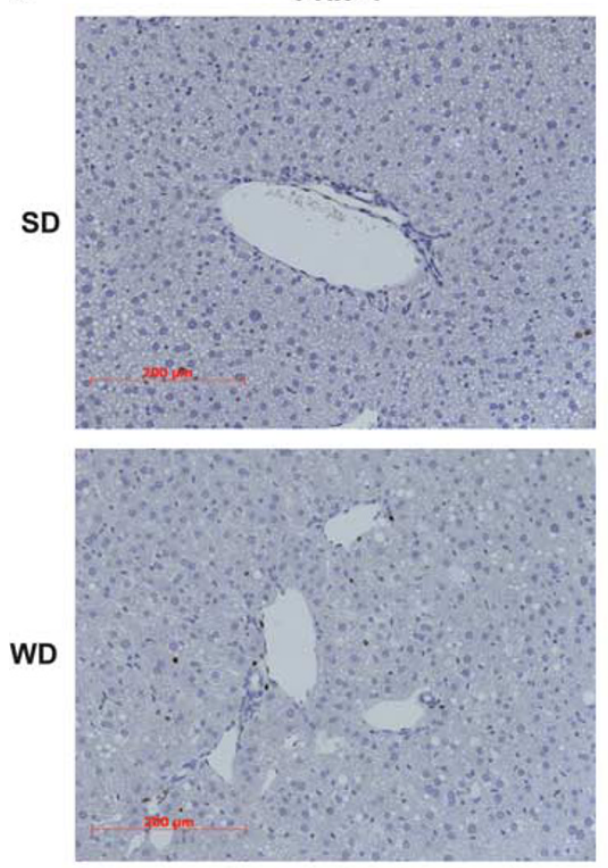

b

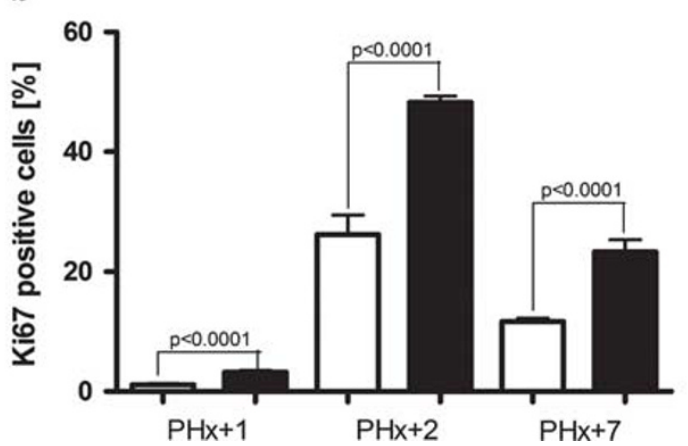

PHx+2
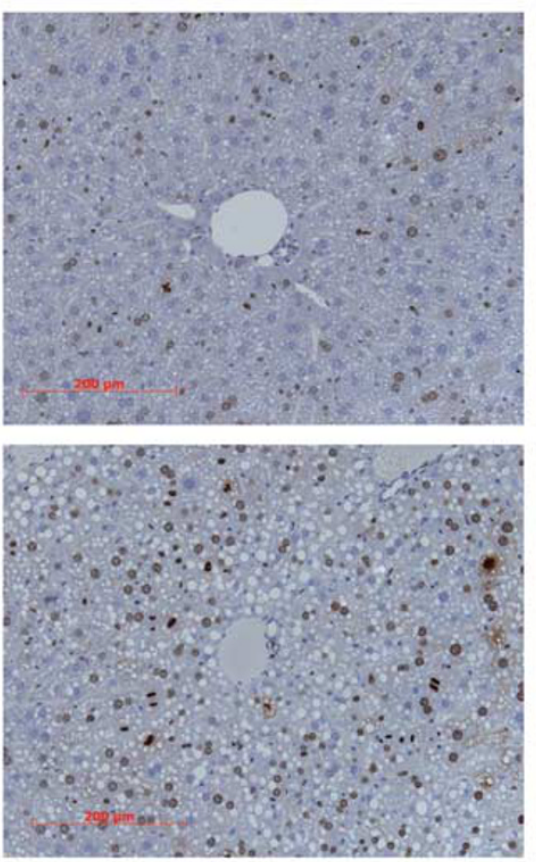

c
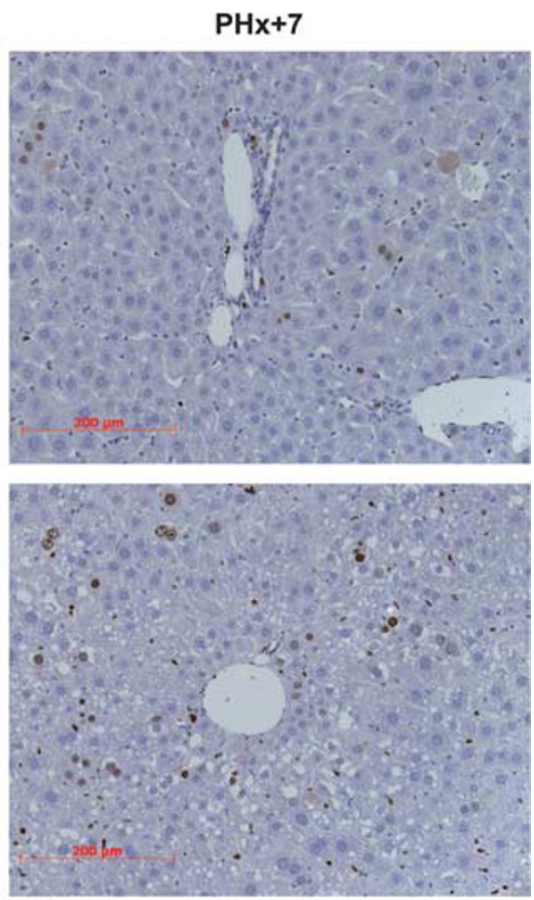

$p<0.0001$

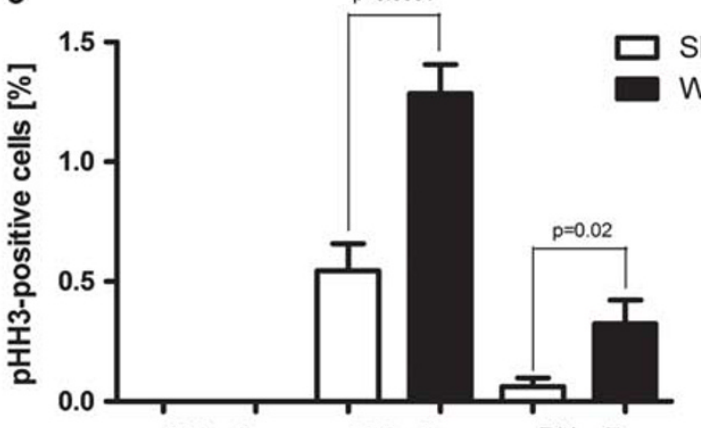

$\mathrm{PHx}+1$

$\mathrm{PHx}+7$

Figure 5 Proliferation of liver cells in a dietary steatosis model after 70\% hepatectomy. Ki67 immunochemistry (a and b) shows that western diet (WD) enhanced the numbers of Ki67 and phospho histone H3-positive cells (c) compared with standard diet (SD)-fed mice, in particular on postoperative days $(\mathrm{PHx}+) 2$ and 7. Quantification shows mean \pm s.e.m. of Ki67-(b) or phospho histone H3-positive cells (c) per total cells (20-fold magnification).

PHx: 70\% hepatectomy; pHH3: phosphor-histone H3.

\section{PHx in Steatotic Livers Induces DR Expression without Induction of Apoptosis}

As we and others have shown previously, DR expression is increased in steatotic livers. It is not known however, if this effect enhances the vulnerability of hepatocytes in liver surgery. Expression levels of DR were notably upregulated after $70 \%$ PHx in WD-fed mice on PHx $+1, \mathrm{PHx}+2$ and $\mathrm{PHx}+7$ (Figure $4 \mathrm{a}$ and $\mathrm{b}$ ). The pro-apoptotic molecule Noxa was upregulated in SD- and WD-fed mice (Figure 4c) with a more prominent increase in the WD groups postoperatively. At the same time, expression of anti-apoptotic molecules $B c l-2, B c l-x l$ and $M c l-1$ was increased on $\mathrm{PHx}+1$ and $\mathrm{PHx}+2$ (Figure $4 \mathrm{~d}-\mathrm{f}$ ), again with a higher increase in WD animals. Evaluation of $B c l-2 / B a x$ ratio demonstrated a surplus of the anti-apoptotic molecule $\mathrm{Bcl}-2$ versus pro-apoptotic Bax and therefore demonstrates a pro-survival potential of the liver cells in WD-fed animals (Figure 4g). Compared with preoperative numbers, TUNEL staining revealed more positive cells after 70\% $\mathrm{PHx}$ in liver tissue, though no significant differences between the two groups were observed (Figure 4h).

\section{0\% PHx Leads to Dephosphorylation of Hgf Downstream Target Erk1/2 in SD-Fed Mice}

To further elucidate the role of $\mathrm{Hgf}$ in the regenerating steatotic liver, we analyzed the phosphorylation status of Erk1/2 (Figure 6a-d), which is a downstream target of $\mathrm{Hgf}$ signaling. SD mice exhibited enhanced Erk1/2 phosphorylation before $\mathrm{PHx}$, which was diminished on $\mathrm{PHx}+1$ and $\mathrm{PHx}+2$. In contrast, Erk1/2 phosphorylation was low in the 

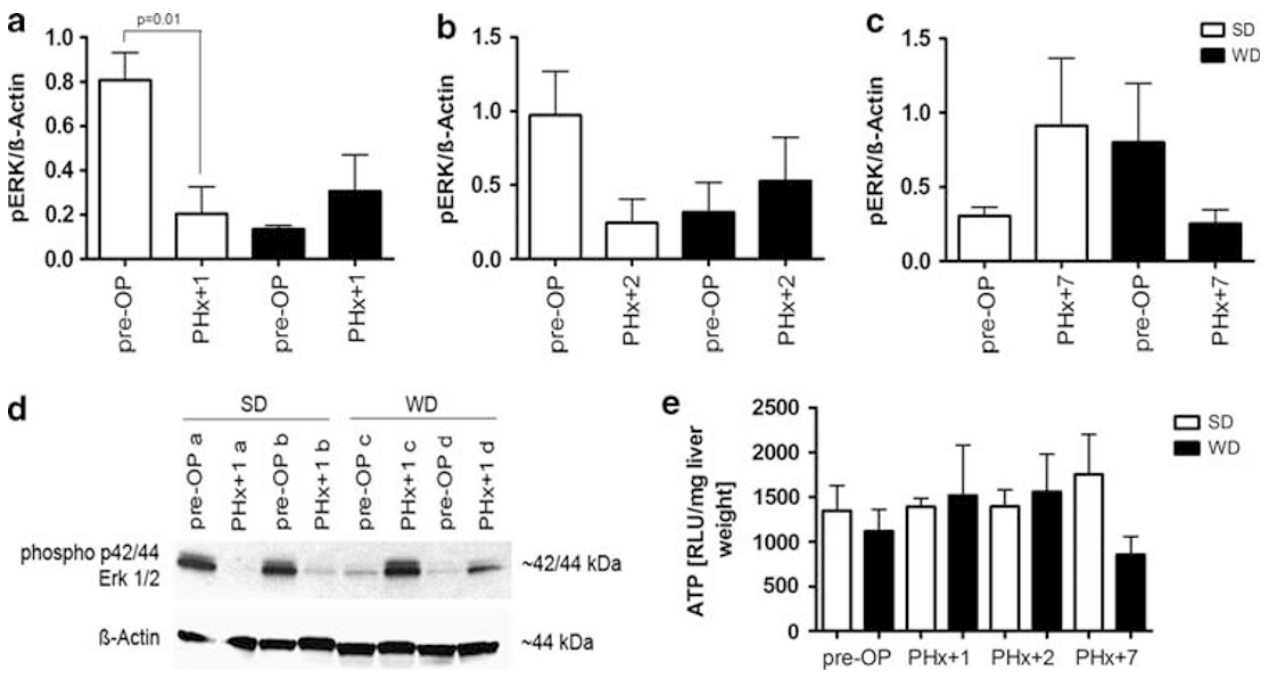

Figure 6 Western blot analysis of Erk1/2 phosphorylation and measurement of ATP in steatotic liver tissue before and after 70\% hepatectomy. $70 \%$ hepatectomy (PHx) causes dephosphorylation of Erk1/2 in regenerating liver tissue in standard diet (SD)-fed mice. The majority of western diet (WD)-fed animals exhibited converse development with enhanced Erk1/2 phosphorylation postoperatively. Quantification of bands detected by phosphoErk1/2 antibody normalized to $\beta$-actin bands on $\mathrm{PHx}+1$ (a), $\mathrm{PHx}+2$ (b) and $\mathrm{PHx}+7$ (c). White bars represent mice fed a standard diet; black bars refer to animals receiving WD. Representative western blot showing two individual mice each, fed SD or WD (d). (Quantification shown of $n=4$ animals per group as relative intensity of bands normalized to $\beta$-actin \pm s.e.m.). ATP levels are depicted as RLU/mg liver tissue and did not show significant differences between SD and WD liver tissue.

majority of WD-fed mice before surgery but increased after PHx, though differences between the groups did not reach significance. As the lower preoperative phosphorylation status of Erk1/2 in WD-fed mice might be due to lower energy supply within the liver tissue, ATP activity in liver tissues of SD- and WD-fed animals was measured. But no significant differences between dietary groups were recognized before $\mathrm{PHx}$ and on $\mathrm{PHx}+1$ and $\mathrm{PHx}+2$. Only on PHx +7 , ATP levels were slightly decreased in liver tissues of WD-fed animals (Figure 6e).

\section{DISCUSSION}

With increasing prevalence of obesity and associated comorbidities, surgical complications linked to the metabolic syndrome are on the rise. The aim of this study was to elucidate the effects of simple steatosis as hepatic manifestation of the metabolic syndrome on liver regeneration. WD feeding for 6 weeks led to hepatic TAG and macrophage accumulation, increased serum FFA in combination with elevated liver enzymes and leptin in serum compared with SD littermates. After $70 \%$ PHx, liver TAG content did not change, though elevated serum FFA levels were observed in both groups on $\mathrm{PHx}+1$ and hepatic lipid accumulation occurred in SD-fed mice. Enhanced liver regeneration was found in WD-fed mice after 70\% PHx, which was associated with DR and FATP upregulation as well as activation of Hgf signaling in association with altered Erk1/2 phosphorylation.

Previous studies have shown that liver regeneration after PHx was accompanied by hepatic fat accumulation caused by increased circulating cytokines stimulating the release of fatty acids from adipose tissue. In the liver, these fatty acids are stored as triacylglycerols within intracellular fat droplets determined for $\beta$ oxidation or reconstruction of cell membranes. ${ }^{13}$ Elevated FATP expression after PHx may thus contribute to FFA uptake in hepatocytes in support of hepatic regeneration. The importance of FATP for liver regeneration has been implied previously, ${ }^{17}$ which is in line with our data, as the already TAG- and fat-laden hepatocytes exhibited earlier and stronger proliferation after PHx. In the opposite mechanisms, blocking hepatic fat accumulation or FABP decreases liver regeneration after PHx. ${ }^{13,18-20}$

We and others have previously published a direct correlation between hepatic steatosis and upregulation of FATP and DR, which is associated with increased vulnerability to cell death and liver damage, a hallmark of NAFLD. ${ }^{1,21}$ Although expression levels of FATP and DR were significantly higher in the WD group and activation of macrophages indicate a slight inflammation, no evidence for enhanced liver cell damage and apoptosis was detected. DR stimulation can not only activate the induction of apoptosis but may also enhance expression of cytokines, growth factors, antiapoptotic and proliferative molecules ${ }^{22,23}$ and is essential for liver regeneration. ${ }^{24}$ For example, TNFR stimulation can activate $\mathrm{nF} \kappa \mathrm{B}$, exerting a proliferative effect ${ }^{25}$ and IL-6 protects against Fas-mediated cell death by increasing antiapoptotic protein expression (cFlar, Bcl-2, Bcl-xl). ${ }^{26}$ Here we found that $c$ Flar and IL-6 expression levels (data not shown) as well as serum levels of different cytokines and chemokines were not changed by WD neither before PHx nor in SD- and WD-fed mice after 70\% PHx. The expression of the 
anti-apoptotic molecules $B c l-2$ and $B c l-x l$ was elevated by feeding WD and even higher after PHx in WD-fed animals. Thus, increased DR expression in WD mice and upregulation of Puma and Noxa may have been countered by parallel activation of anti-apoptotic proteins, such as $B c l-2, B c l-x l$ and $M c l-1$, known to suppress apoptosis induction. ${ }^{27}$ Determination of $B c l-2 / B a x$ ratio could show that the rate of the antiapoptotic molecule $B c l-2$ was in excess of the pro-apoptotic molecule Bax, suggesting an overall pro-survival status of the cells.

Steatotic mouse and rat models induced by various disorders in fat and glucose metabolism show impaired and decreased liver regeneration. ${ }^{13,28-33}$ In contrast, solely a mild hepatic inflammation and no evidence of enhanced cell death or the development of insulin resistance was found in our model. Neither glucose nor insulin levels were altered and no relevant differences indicating development of diabetes in our model was observed in the diabetes assay. Moreover, dietary-induced steatosis was associated with elevated cell proliferation after $70 \% \mathrm{PHx}$ compared with normal liver tissue. The limited time of feeding and the composition of high fat/high calorie diet may account for a rather mild steatosis and inflammation, beneficial to regeneration. Other models elicit steatosis, accompanied by manifest inflammation or severe cell damage. A novel dietary model caused characteristics similar to our model, including microvesicular steatosis but without evidence of severe inflammation after receiving a fast food diet, ${ }^{34}$ though no data on resection or regeneration are available for this model yet. In our model, 6 weeks of WD feeding did not change cytokine release or induce severe inflammation and hepatic cell death before and after PHx.

Severe consequences occur in genetic mouse models with deficient leptin signaling, metabolic syndrome or insulin resistance, resulting in increased mortality after $\mathrm{PHx}$ and impaired liver regeneration in surviving animals. ${ }^{29-32}$ In contrast, in our model no insulin resistance was observed under WD feeding and leptin levels were elevated before surgical intervention, which is a hallmark of human obesity. ${ }^{35}$ Leptin, which is a factor released from adipose tissue, may also have an important role within hepatic regeneration and can act pro-proliferative by activating Erk1/2. ${ }^{35-37}$ Additionally, $\mathrm{Hgf} / \mathrm{c}-\mathrm{met}$ signaling is essential for liver regeneration, ${ }^{38-41}$ having a crucial role by activating the MEK/Erk pathway. Upon activation by various stress factors or cytokines, Erk is phosphorylated at threonine and tyrosine residues. Cell-cycle progression and regenerative mechanisms are controlled by Erk activity via suppressing apoptosis and regulation of DNA replication. ${ }^{42,43}$ Our findings demonstrate elevated serum leptin and increased Hgf expression, associated with an altered Erk1/2 phosphorylation pattern in WD-fed mice after PHx. Whereas SD-fed mice showed strong Erk1/2 phosphorylation preoperatively diminishing on $\mathrm{PHx}+1$ and $\mathrm{PHx}+2$, phosphorylated Erk1/2 was only marginally present in WD mice before surgery and increased after surgery. This suggests a mechanism of enhanced hepatocyte proliferation mediated by Hgf signaling, possibly in concert with raised leptin levels, and subsequent Erk1/2 activation.

Many authors suppose that not steatosis itself impairs liver regeneration, but metabolic disorders, serious hepatic inflammation and liver damage may disturb proper liver regeneration..$^{29,44-47}$ In $o b / o b$ mice, for example, hepatocytes are arrested in $G_{1}$ after PHx due to reduced ATP levels. ${ }^{32}$ Therefore, energy supply in the WD model was analyzed, though no alterations in ATP content were observable between SD and WD feeding.

Here, we could show that feeding WD for 6 weeks enhances liver regeneration after 70\% PHx. This was accompanied by elevated expression of DR and FATP as well as increased leptin levels, $H g f$ and Erk1/2 signaling. WD-fed mice were not overly susceptible to liver cell death or hepatic injury, which might be due to elevated expression of antiapoptotic and proliferative molecules. Further experiments are needed to elucidate the exact mechanisms involved in the pro-proliferative effect of mild steatosis as shown here.

Supplementary Information accompanies the paper on the Laboratory Investigation website (http://www.laboratoryinvestigation.org)

\section{ACKNOWLEDGEMENTS}

We thank Lena Wingerter and Dorothe Möllmann for their technical assistance and supply of sections for the various conducted staining methods. This work was supported by the Deutsche Forschungsgemeinschaft (DFG, Grant CA 267/6-1 part of KFO-117 and CA 267/8-1) and the Wilhelm Laupitz Foundation to AC.

\section{DISCLOSURE/CONFLICT OF INTEREST}

The authors declare no conflict of interest.

1. Bechmann LP, Gieseler RK, Sowa J-P, et al. Apoptosis is associated with CD36/fatty acid translocase upregulation in non-alcoholic steatohepatitis. Liver Int 2010;30:850-859.

2. Angulo P. Nonalcoholic fatty liver disease and liver transplantation. Liver Transpl 2006;12:523-534.

3. Doege $H$, Grimm D, Falcon A, et al. Silencing of hepatic fatty acid transporter protein 5 in vivo reverses diet-induced non-alcoholic fatty liver disease and improves hyperglycemia. J Biol Chem 2008;283: 22186-22192.

4. Pohl J, Ring A, Korkmaz U, et al. FAT/CD36-mediated long-chain fatty acid uptake in adipocytes requires plasma membrane rafts. Mol Biol Cell 2005;16:24-31.

5. Brookheart RT, Michel $\mathrm{Cl}$, Schaffer JE. As a matter of fat. Cell Metab 2009;10:9-12.

6. Selzner M, Clavien P-A. Fatty liver in liver transplantation and surgery. Semin Liver Dis 2001;21:105-114.

7. McCormack L, Dutkowski P, El-Badry AM, et al. Liver transplantation using fatty livers: always feasible?. J Hepatol 2010;54:1055-1062.

8. Bellentani $\mathrm{S}$, Scaglioni $\mathrm{F}$, Marino $\mathrm{M}$, et al. Epidemiology of nonalcoholic fatty liver disease. Dig Dis 2010;28:155-161.

9. Vanni E, Bugianesi $E$, Kotronen A, et al. From the metabolic syndrome to NAFLD or vice versa?. Dig Liver Dis 2010;42:320-330.

10. Fishbein TM, Fiel MI, Emre S, et al. Use of livers with microvesicular fat safely expands the donor pool. Transplantation 1997;64:248-251.

11. Cho JY, Suh K-S, Kwon CH, et al. Mild hepatic steatosis is not a major risk factor for hepatectomy and regenerative power is not impaired. Surgery 2006;139:508-515. 
12. Shteyer E, Liao Y, Muglia LJ, et al. Disruption of hepatic adipogenesis is associated with impaired liver regeneration in mice. Hepatology 2004:40:1322-1332.

13. Newberry EP, Kennedy SM, Xie Y, et al. Altered hepatic triglyceride content after partial hepatectomy without impaired liver regeneration in multiple murine genetic models. Hepatology 2008;48:1097-1105.

14. Tijburg LB, Nyathi CB, Meijer GW, et al. Biosynthesis and secretion of triacylglycerol in rat liver after partial hepatectomy. Biochem J 1991;277:723-728.

15. Debonera F, Aldeguer X, Shen X, et al. Activation of interleukin-6/ STAT3 and liver regeneration following transplantation. J Surg Res 2001;96:289-295.

16. Higgins G, Andersson T. Experimental pathology of the liver: 1 . Restoration of the liver of the white rat following partial surgical removal. Arch Pathol 1931;12.

17. Wang G, Chen QM, Minuk GY, et al. Enhanced expression of cytosolic fatty acid binding protein and fatty acid uptake during liver regeneration in rats. Mol Cell Biochem 2004;262:41-49.

18. Fernández MA, Albor $C$, Ingelmo-Torres $M$, et al. Caveolin-1 is essential for liver regeneration. Science 2006;313:1628-1632.

19. Brasaemle DL. A metabolic push to proliferate. Science 2006;313: 1581-1582.

20. Farrell GC, Robertson GR, Leclercq I, et al. Liver regeneration in obese mice with fatty livers: does the impairment have relevance for other types of fatty liver disease?. Hepatology 2002;35:731-731.

21. Feldstein $A E$, Canbay $A$, Guicciardi $M E$, et al. Diet associated hepatic steatosis sensitizes to Fas mediated liver injury in mice. J Hepatol 2003;39:978-983.

22. Kirillova I, Chaisson M, Fausto N. Tumor necrosis factor induces DNA replication in hepatic cells through nuclear factor kappaB activation. Cell Growth Differ 1999;10:819-828.

23. Bechmann LP, Zahn D, Gieseler RK, et al. Resveratrol amplifies profibrogenic effects of free fatty acids on human hepatic stellate cells. Hepatol Res 2009;39:601-608.

24. Yamada Y, Kirillova I, Peschon JJ, et al. Initiation of liver growth by tumor necrosis factor: deficient liver regeneration in mice lacking type I tumor necrosis factor receptor. Proc Natl Acad Sci USA 1997;94:1441-1446.

25. Chaisson ML, Brooling JT, Ladiges W, et al. Hepatocyte-specific inhibition of NF-kappaB leads to apoptosis after TNF treatment, but not after partial hepatectomy. J Clin Invest 2002;110:193-202.

26. Kovalovich K, Li W, DeAngelis $\mathrm{R}$, et al. Interleukin-6 protects against Fas-mediated death by establishing a critical level of anti-apoptotic hepatic proteins FLIP, Bcl-2, and Bcl-xL. J Biol Chem 2001;276:2660526613.

27. Tzung SP, Fausto N, Hockenbery DM. Expression of $\mathrm{Bcl}-2$ family during liver regeneration and identification of $\mathrm{BCl}-\mathrm{x}$ as a delayed early response gene. Am J Pathol 1997;150:1985-1995.

28. Anderson SP, Yoon L, Richard EB, et al. Delayed liver regeneration in peroxisome proliferator-activated receptor-alpha-null mice. Hepatology 2002;36:544-554.

29. Leclercq IA, Vansteenberghe M, Lebrun VB, et al. Defective hepatic regeneration after partial hepatectomy in leptin-deficient mice is not rescued by exogenous leptin. Lab Invest 2006;86:1161-1171.
30. Yamauchi $\mathrm{H}$, Uetsuka $\mathrm{K}$, Okada $\mathrm{T}$, et al. Impaired liver regeneration after partial hepatectomy in $\mathrm{db} / \mathrm{db}$ mice. Exp Toxicol Pathol 2003; 54:281-286.

31. Ezaki H, Yoshida Y, Saji Y, et al. Delayed liver regeneration after partial hepatectomy in adiponectin knockout mice. Biochem Biophys Res Commun 2009;378:68-72.

32. Yang S. Disrupted signaling and inhibited regeneration in obese mice with fatty livers: Implications for nonalcoholic fatty liver disease pathophysiology. Hepatology 2001;34:694-706.

33. Bechmann LP, Hannivoort RA, Gerken G, et al. The interaction of hepatic lipid and glucose metabolism in liver diseases. J Hepatol 2012;56:952-964.

34. Charlton M, Krishnan A, Viker $\mathrm{K}$, et al. Fast food diet mouse: novel small animal model of NASH with ballooning, progressive fibrosis, and high physiological fidelity to the human condition. Am J Physiol Gastrointest Liver Physiol 2011;301:G825-G834.

35. Catalano S, Mauro L, Marsico S, et al. Leptin induces, via ERK1/ERK2 signal, functional activation of estrogen receptor alpha in MCF-7 cells. J Biol Chem 2004;279:19908-19915.

36. Liu Y, Lv L, Xiao W, et al. Leptin activates STAT3 and ERK1/2 pathways and induces endometrial cancer cell proliferation. J Huazhong Univ Sci Technol Med Sci 2011;31:365-370.

37. Wree A, Kahraman A, Gerken G, et al. Obesity affects the liver-the link between adipocytes and hepatocytes. Digestion 2011;83:124-133.

38. Ogura Y, Hamanoue M, Tanabe G, et al. Hepatocyte growth facto promotes liver regeneration and protein synthesis after hepatectomy in cirrhotic rats. Hepatogastroenterology 2001;48:545-549.

39. Nakamura T. Hepatocyte growth factor as mitogen, motogen and morphogen, and its roles in organ regeneration. Int Symp Princess Takamatsu Cancer Res Fund 1994;24:195-213.

40. Huh C-G, Factor VM, Sánchez A, et al. Hepatocyte growth factor/c-met signaling pathway is required for efficient liver regeneration and repair. Proc Natl Acad Sci USA 2004;101:4477-4482.

41. Borowiak M, Garratt AN, Wüstefeld $T$, et al. Met provides essential signals for liver regeneration. Proc Natl Acad Sci USA 2004;101: 10608-10613.

42. Talarmin H, Rescan C, Cariou S, et al. The mitogen-activated protein kinase kinase/extracellular signal-regulated kinase cascade activation is a key signalling pathway involved in the regulation of $\mathrm{G}(1)$ phase progression in proliferating hepatocytes. Mol Cell Biol 1999;19:6003-6011.

43. Campbell JS, Argast GM, Yuen SY, et al. Inactivation of p38 MAPK during liver regeneration. Int J Biochem Cell Biol 2011;43:180-188.

44. Rao MS, Papreddy K, Abecassis M, et al. Regeneration of liver with marked fatty change following partial hepatectomy in rats. Dig Dis Sci 2001;46:1821-1826.

45. Picard C, Lambotte L, Starkel P, et al. Steatosis is not sufficient to cause an impaired regenerative response after partial hepatectomy in rats. J Hepatol 2002;36:645-652.

46. Clavien P-A, Oberkofler CE, Raptis DA, et al. What is critical for liver surgery and partial liver transplantation: Size or quality?. Hepatology 2010;52:715-729.

47. Tsai $\mathrm{C}-\mathrm{Y}$, Lin $\mathrm{Y}-\mathrm{S}$, Yeh T-S, et al. Disrupted hepatic adiponectin signaling impairs liver regeneration of steatotic rats. Chang Gung Med J 2011;34:248-259. 